\title{
An Efficient Participant's Selection Algorithm for Crowdsensing
}

\author{
*Tariq Ali,Umar Draz, Sana Yasin, Javeria Noureen, Ahmad shaf \\ CS. Department \\ (CIIT) Sahiwal, Pakistan
}

\author{
Munwar Ali \\ CS. Department \\ (CIIT) Lahore, Pakistan
}

\begin{abstract}
With the advancement of mobile technology the use of Smartphone is greatly increased. Everyone has the mobile phones and it becomes the necessity of life. Today, smart devices are flooding the internet data at every time and in any form that cause the mobile crowdsensing (MCS). One of the key challenges in mobile crowd sensing system is how to effectively identify and select the well-suited participants in recruitments from a large user pool. This research work presents the concept of crowdsensing along with the selection process of participants from a large user pool. MCS provides the efficient selection process for participants that how well suited participant's selects/recruit from a large user pool. For this, the proposed selection algorithm plays our role in which the recruitment of participants takes place with the availability status from the large user pool. At the end, the graphical result presented with the suitable location of the participants and their time slot.
\end{abstract}

Keywords-Mobile crowdsensing (MCS); Mobile Sensing Platform (MSP]); crowd sensing; participant; user pool; crowdsourcing

\section{INTRODUCTION}

Today a mobile phone is the essential part of life. The use of mobile phones has greatly increased; the latest mobile phones now come with many embedded sensors. The capabilities of mobile phones have been greatly increased in the recent years, for instance, processing power, embedded sensors, storage capacities and network information rates [1]. This advancement of technologies combined with the huge number of client companioned cell phones empowers another and quickly developing sensing paradigm called Crowdsensing. Crowdsensing is the ability by which application developers can make tasks and recruit cell phone clients to give sensor information to be utilized towards a particular goal. Crowdsensing is also sometimes referred as a mobile Crowdsensing. A formal way to represent the mobile Crowdsensing (MCS) is: Mobile Crowdsensing (MCS) presents a new sensing model, which is based on the power of mobile devices. The absolute number of user companioned devices such as mobile phones, wearable devices, and smart vehicles so on [2], and their inherent mobility empowers a new and fast-growing.

Mobile Crowdsensing (MCS) awards a tremendous measure of wireless customers that offer neighborhood learning (e.g., nearby data, encompassing setting, calmer level, and activity conditions) accumulated by their sensor-improved contraptions. Mobile phone use for computation and acquires a richer functionality. It has a variety of sensors such as camera, microphone, Global Positioning System, accelerometer etc.
Health and pollution monitoring sensors will be intended in the coming future. Mobile sensors such as smartphones and vehicular systems represent a new type of geographically distributed sensing infrastructure that enables mobile peoplecentric sensing. Until recently mobile sensing research such as activity recognition, where peoples activity (e.g., walking, driving, sitting, talking) is classified and monitored, required specialized mobile devices (e.g., the Mobile Sensing Platform [MSP]) [3]. Crowdsensing have diverse applications which are separated into three classifications:

1) Framework checking.

2) Individual to individual correspondence checking.

3) Natural checking.

During a few years ago, a mobile device has been explored to contribute project- 4 that continuously reported the total number of examined birds surrounding the US. Another measurement is Noise pollution [4] is the worst issue in the world. There are so many problems with noise pollution. These problems further affect the standard of health and life. Many diseases are being a part of noise pollution like blood pressure and so on [5]. The European Union commission is advised the other country to control the noisy areas through environmentfriendly sensors [6]. To control the noise pollution in the city sensing nodes has been deploying by several governments through the noisy areas. With the help of noise map that visualizes the graphical view about those areas where the amount of sound level distribution is high. To measure the environmental noise some noise tube system also proposed [3] . Main architecture of mobile crowd sensing is in Fig. 1.

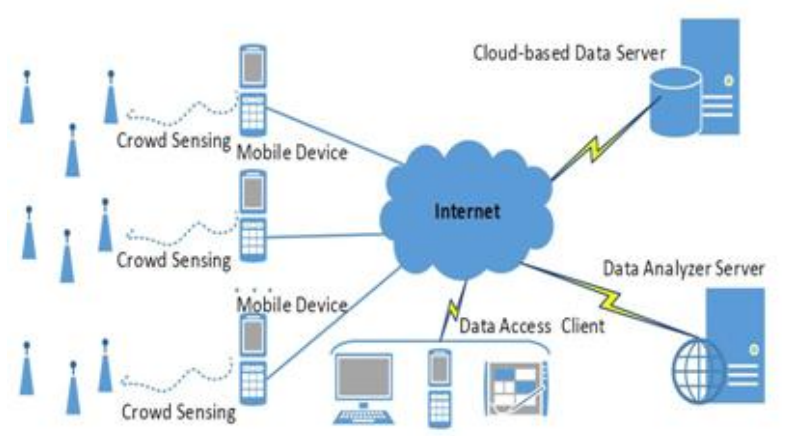

Fig. 1. Architecture of MCS.

This paper presents the selection algorithm of participants, that how participants select to perform a task from a large user 
pool. Participants recruit on the base of availability to minimize the cost of sensing task. Section II describes the related work that was purposed on the crowd sensings parameters and its income challenges. In Sections III and IV, the selection criteria and selection algorithm are discussed. Results and conclusion are written in Sections V and VI, respectively.

\section{RELATED WORK}

The growing sensing capabilities of smartphones have gone past the sensor systems concentrate on environmental and infrastructure monitoring. Individuals are currently the bearers of sensing devices and the sources and consumers about the sensed events [7]. Mobile Crowdsensing plays an analogous role with the one played through Amazons Mechanical Turk (MTurk) or ChaCha in crowdsourcing [8]. It permits individuals and organizations (clients) to access an absolute number of people (providers) ready to execute simple sensing tasks for which they are paid. Unlike the MTurks tasks which are executed on personal computers and always need human work. Mobile sensing tasks are accomplished on mobile devices that fulfill particular context/sensing requirements (e.g., location, time, particular sensors) and sometimes do not need human work (i.e., automatic sensing tasks [9].

Smartphones already have numerous sensors like a camera; microphone, GPS, accelerometer and in the near future they are intended to include health and pollution monitoring sensors. Vehicular systems [10] have access to numerous hundred sensors embedded in cars, and latest vehicles come equipped with new types of sensors, for example, radar and camera. It has been believing that researchers in a number of fields of science and engineering as well as local state, and federal agencies can significantly benefit from this new sensing infrastructure as they will have access to valued data from the physical world. Moreover, commercial organizations might be very interested in gathering mobile sensing data to get more about customer behavior. The participants in mobile crowd sensing systems may need significant incentives to go out of their way and cover out of favor regions. In author situation, they give the complete detail in Biketastic that provide the incentives to participants through sharing bicycle ride. Another assorted quality of motivation is allowing information dealing to get extra data, for instance, deal chasing by means of value questions in Live Compare [11].

\section{A. Crowdsensing Challenges}

Crowdsensing has many challenges here discussed some privacy and security challenges, issues and limitation for mobile crowd sensing [12]. Neighbourhood examination is entering the challenge of finding heuristics and outlining calculations thats whole the pretend meaning. Similar examples of this function are reducing or eliminating the noise and cover the gaps of data. For example, GPS test can't have the capacity to get right or missing, in this time anomalies must be taking out excluded tests extrapolated. The 3-level structure building also have a couple of troubles are according to the accompanying: (a) simulate a computing, (b) arrangement and execution trial between virtual machines correspondences, (c) Correspondence execution is about near to the ground at what time stand out from between process correspondence [13]. Movement affected Reconfiguration is another challenge like
MoneyBee. By using different model and systems most of the MCS produce the same type of data. To make the MCS as an honest application the problem is what are the possible ways in which provide the true and essential information in which true contribution takes place. The arrangement is recent, various amusement hypothesis approaches have been proposed for versatile group detecting and registering to empower and compensate honest commitments. For a unique versatile group detecting and registering framework, there is still a requirement for new motivation and estimating components to draw in, move, and reward honest and excellent detecting information givers. For information conveyance, data conveyance in the transient system is additionally challenged in portable group detecting, how to dispatch the detected information from appropriated members. With the help of host, it can detect the account and its arrangement. versatile group detecting, figuring attributes, instance transfer speed, remote correspondence, repetitive system allotting because of human portability, and a colossal number of vitality obliged gadgets. Planning calculations can comprehend this inconvenience and utilized detecting servers to orchestrate detecting occasions of cell phones (a motivation instrument utilize selected). Note that shrewd detecting applications will just utilize the planning calculations.

1) Crowed sensing privacy: Assurance of privacy is basic for everyone. No one needs to reveal his/her security before anyone. In the current system use unmistakable systems to offer security to PDAs or center points. Privacy mechanism is responsible to provide security defending segments to data supporters. Some part, for example, assignment distribution, sensor doors, information anonymization, motivation component and huge information stockpiling are utilized as a part of this layer, which gathered information from the chose hubs [4], [14], [15]. Most famous privacy approaches are discussed in [16]:

1) Pseudonyms: It is the straightforward method that makes members unknown by supplanting their recognizable proof data with an assumed name.

2) Connection anonymization.

Utilizing this method, we can keep away from the system based following assaults utilizing IP addresses. One such procedure which is utilized as a part of Crowd detecting applications is onion steering. Another protection safeguarding approach in which some calligraphy techniques are used. Moreover, some essentialness usage also has been noticed in the mobile crowdsensing [17]. The k-namelessness procedure can be connected keeping in mind the end goal to give the area security of the members who transfer reports. The essential thought behind the k-namelessness method develop gatherings of ' $\mathrm{N}$ ' members. Along these lines normal trait sharing, (like 'N' members arranged each district), translating then indistinguishable each other. To construct a gathering of ' $k$ ' clients it can utilize diverse strategies to locate the appropriate and basic property. So these techniques ordered into two fundamental segments, for example, speculation and bother. With the wide adoption of mobile Crowdsensing applications, task coverage and participant selection in MCS systems have captured the attention of researchers. First, there are several systems and experimental studies on either experimental study on MCS coverage or general framework of participant recruitment [18], 
[19]. For example in [20] has performed a systematic study of the coverage and scaling properties of place-centric urban crowd sensing and shows promising results that MCS can provide relatively high coverage levels especially given area with large size. Then, there are also many theoretical studies on various task assignment and participant selection problems, playing tradeoffs among sensing cost, task coverage, energy efficiency [21], [22] and user privacy [23], and incentive. In offline study participant selection in the piggyback mechanism, in which MCS for probabilistic coverage at this situation so that task is easily performed. They aim to select a minimum number of participants to guarantee the selected participants will make enough number of calls at a certain percentage of the target locations over a long-fixed sensing period. Protection, security, difficulties, and hazard that uncovers the delicate information about members with respect to privacy is easily be solved if the well-suited participants need to select from the large user pool. We will concentrate on the social and specialized difficulties or dangers. Fig. 2 is represented a generic structure of task flow in MCS.

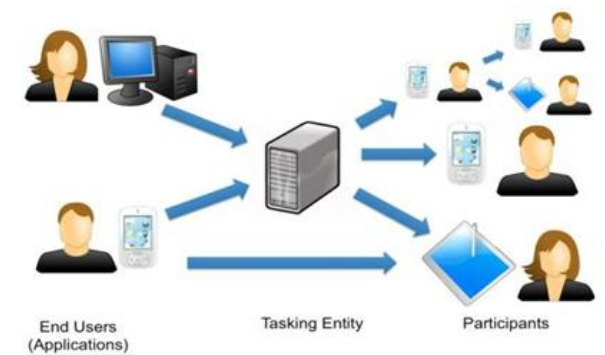

Fig. 2. Generic structure of task flow in MCS.

In this section, a different concept is presented such as Crowdsensing, Crowdsensing application, Crowdsensing challenges and privacy issues and Crowdsensing related work. MCS allows the extensive measure of mobile phone customers share local learning, for example; (nearby data, encompassing setting). Crowdsensing has many challenges some privacy and security challenges are mentioned in [16]. To get the privacy different privacy techniques can use to protect the user privacy such as Anonymization, encryption and data perturbation but still, there is some privacy threats need to be solved. The key challenge which identity in this research work is how to effectively identify the well-suited participant from the large user pool [17]. The recruitment process becomes more complicated when the sensing tasks are dynamic and heterogeneous.

\section{SELECTION CRITERIA FOR PARTICIPANTS}

In selection algorithm, it is assumed that ' $\mathrm{N}$ ' number of participants has been recruited. ' $\mathrm{N}$ ' number of participants selects from the user pool and user pool is divided into two partitions. Selection is done by the parallel searching to check the availability of participants. Design algorithm uses two values ' 0 ' and ' 1 ' in two locations. If the value is $0-1$ then the participant is selected same as in the 1-0. If 0-0 in both locations then participants will not be select. Table I shows the availability of participants.
TABLE I. PARTICIPANTS RAKING

\begin{tabular}{|c|c|c|c|c|c|c|c|c|c|c|}
\hline Users & 1 & 2 & 3 & 4 & 5 & 6 & 7 & 8 & 9 & 10 \\
\hline L1 & 0 & 0 & 1 & 1 & 0 & 0 & 1 & 1 & 0 & 0 \\
\hline L2 & 1 & 1 & 0 & 0 & 0 & 0 & 0 & 0 & 1 & 1 \\
\hline
\end{tabular}

\section{Selection Algorithm}

In this algorithm, well-suited participants are selected from a large user pool. User pool divides into two segments that parallel check and selects the participants this increase the efficiency of the algorithm. For example, we have two locations L1 and L2. For the selection of participants, it is necessary that participants should be available either in L1 or L2. If participant available in any one location it will be selected otherwise it will not be selected.

\section{A. Pseudo Code}

In this algorithm, user pool is divided into two segments such as $\frac{x}{2}$. ' $\mathrm{X}$ ' represents the rang of user pool.

\begin{tabular}{l}
\hline Algorithm 1 Selection algorithm \\
\hline 1: Start \\
2: Input: N numbers of participants \\
3: Output: Selected participants. \\
4: $a$ is user pool // takes input from user pool \\
5: $s$ elected $=0 / /$ initialize the variable (selected) from zero \\
6: $u$ nselected =0 // initialize the variable (unselected) from \\
zero
\end{tabular}

\section{Segment 1}

7: for ( $r$ in 1: $m$ id) // this loop runs from 1 to mid for selecting users from the first segment of user pool

8: do

9: for $(u$ in 1:2) // inner for loop is running from 1 to 2 times used for locations

10: do

11: $\quad k=1$

12: if $a[r, k]==1$ OR $a[r, k=\mathrm{k}+1]==1$ then

13: $\quad$ selected $=$ selected $+1 / /$ counts the selected participants from segment 1

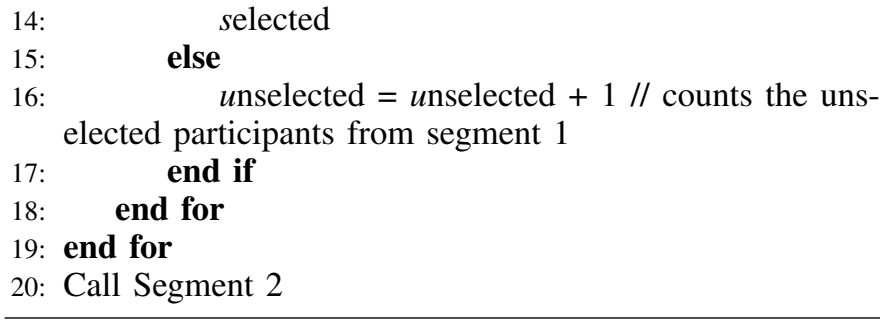

\section{B. Description of Pseudo Code}

In this algorithm, participants are selected from a large user pool. To increase the efficiency of this algorithm user pool is divided into two partitions $(\mathrm{x} / 2)$ that efficiently selects the participants from the user pool. There are ' $\mathrm{x}$ ' time slots and two locations L1 and L2. 
Segment 2

21: for ( $p$ in mid $: x$ ) // this loop runs from mid to $\mathrm{x}$ that checks the second segment of the user pool for selecting participants

22: do

23: for ( $t$ in 1:2) // inner for loop is running from 1 to 2 times used for locations

24: do

25: $\quad q=1$

26: $\quad$ if $a[p, q]==1$ OR $a[p, q=\mathrm{q}+1]==1$ then

27: $\quad$ selected $=$ selected $+1 / /$ counts the selected participants from segment 2

28: $\quad$ selected

29: $\quad$ else

30: $\quad u$ nselected $=u$ nselected $+1 / /$ counts the uns-

elected participants from segment 2

31: $\quad$ end if

32: $\quad$ end for

33: end for

34: print ("overall selected and unselected users from user pool")

35: selected // counts the total number of selected users from pool

36: $u$ nselected // counts the total number of unselected users from pool

37: End

1) Variables initialization

Selected $=0$

Unselected $=0$

2) Loops:

Two loops are used in this algorithm. The first loop runs from 1 to mid for selecting users from the first segment of user pool. The second loop is running from 1 to 2 used for locations.

3) Conditions:

Assign value to $\mathrm{k}=1$ and use condition if $(\mathrm{a}[\mathrm{r}, \mathrm{k}]==1$ OR a $[\mathrm{r}, \mathrm{k}=\mathrm{k}+1]==1$ ). In this condition can specify as a(user pool), r(used for loop to check the participants), $\mathrm{k}$ (it checks the availability of participants).

- If else condition

A conditional statement is used to check the availability of participants.

- If $\mathrm{k}$ finds 1 in any location it means that conditions are true then add 1 in a selected variable such as selected $=$ selected +1 . All selected participants value stored in a selected variable. This condition will run until a condition is false.

Else if $\mathrm{k}$ finds zero in both locations, then the participant is not available and add 1 in the unselected variable such as unselected=unselected +1 .

The above process will repeat for the second partition of user pool. At the end, print the total number of selected and unselected participants from the user pool.

\section{Working of Selection Algorithm}

In Fig. 3 flowchart of overall working of the selection, algorithm is shown.

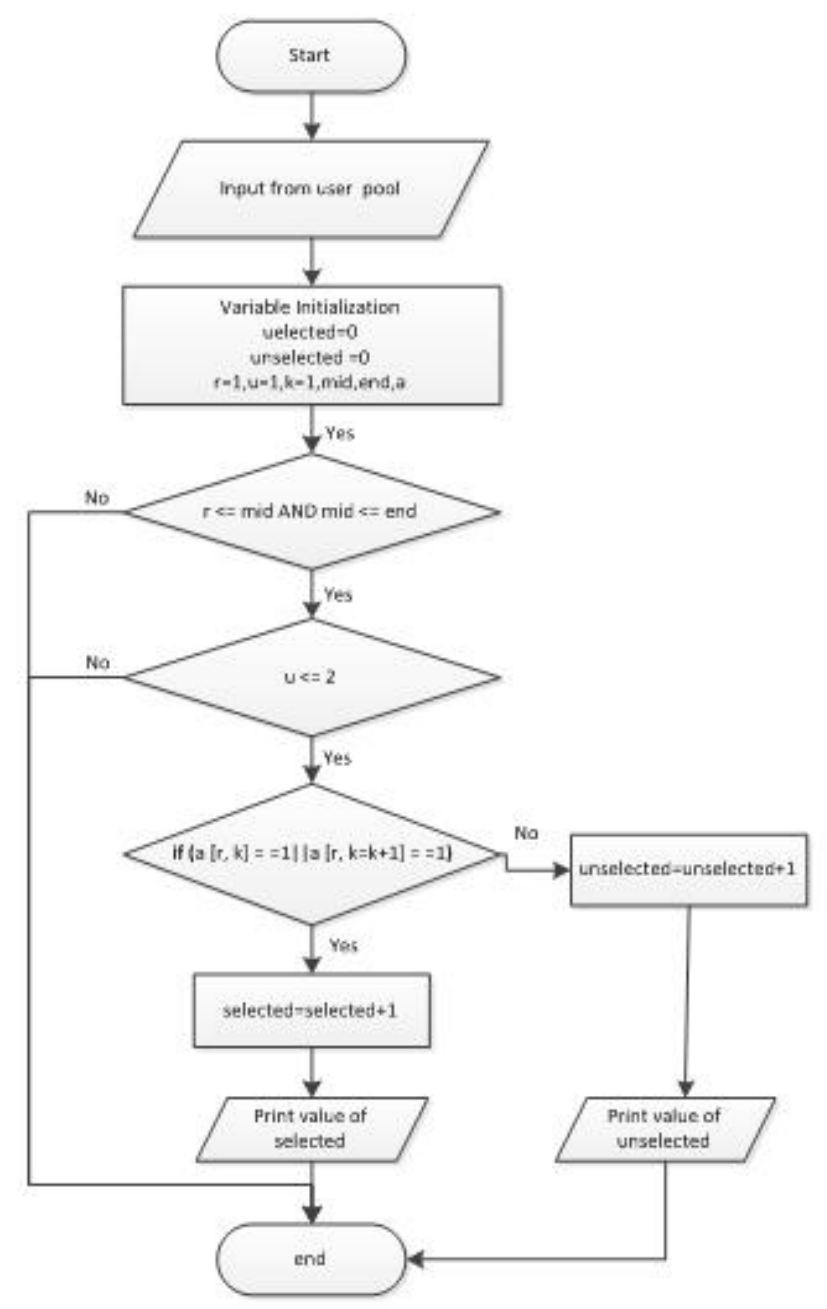

Fig. 3. Flowchart of selection algorithm.

\section{RESULTS}

The selection algorithm of participants from a large user pool on the base of their availability is successfully implemented. User pool divides into two segments that parallel check and selects the participants. If the participants are available in the location, they will be selected to participate in the user pool. Otherwise, they will not be selected. To participate in the user pool, the participants must be selected. In another case, participant will not be eligible to participate. So here we can see how participants selected for the participation. It is assumed that user pool range is 12 and $12 / 2=6$. The design algorithm parallels check both partitions of user pool for the selection of participants. This Fig. 4 shows that 10 participants are willing to participate in the user pool.

\section{A. Graphical Representation of Results}

This section shows the graphical representations are as follows as in Fig. 5. 


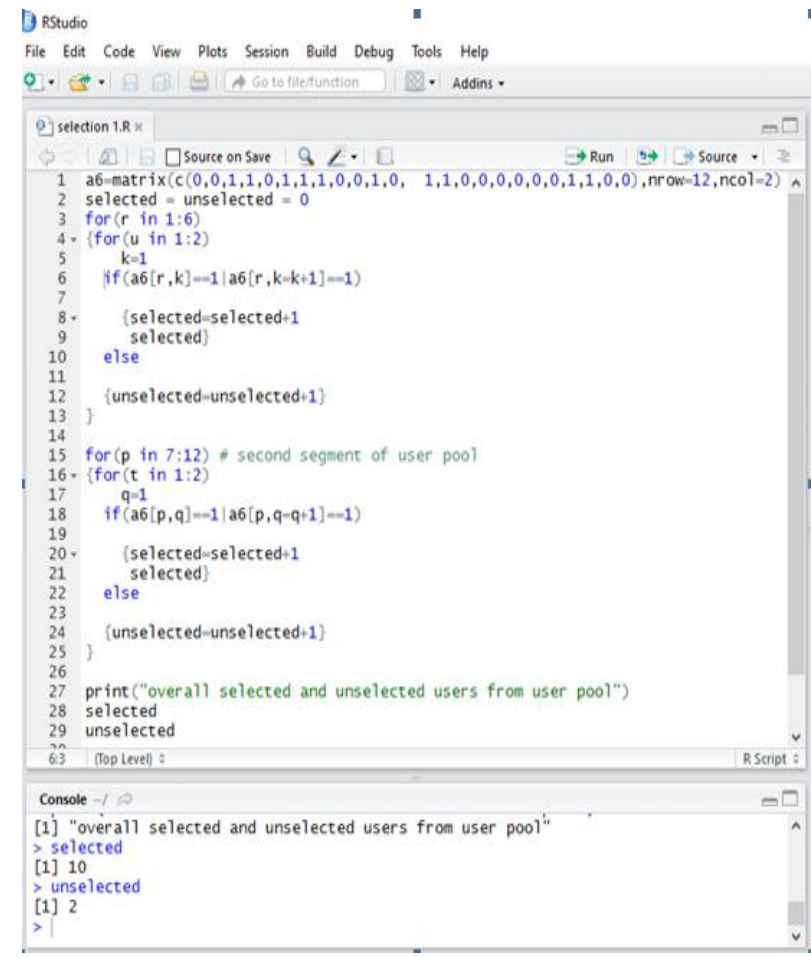

Fig. 4. Selection of participants.

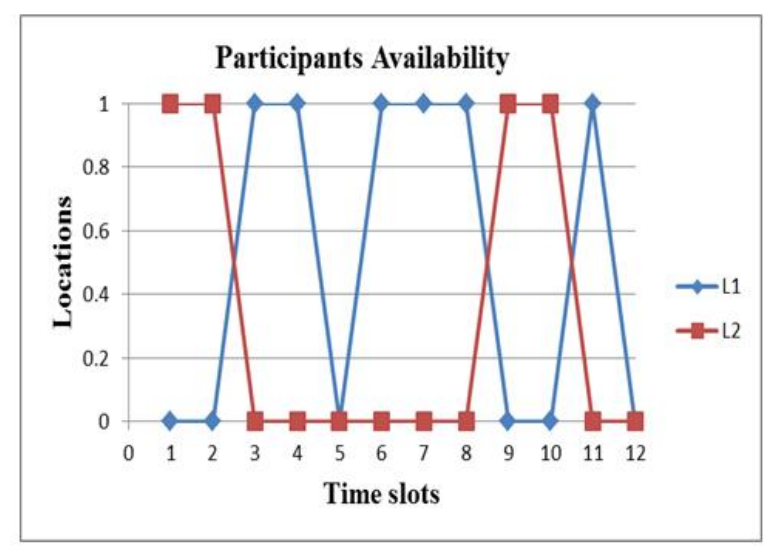

Fig. 5. Participant's availability.

Fig. 5 presents the availability of participants based on values as shown in Table I. From the figure at value 5 and 12 , there is no participants are available and other points show the availability of participants. ' $L$ ' represents the locations.

\section{CONCLUSION}

In this research work, literature is reviewed to understand the dimensions related to ongoing and emerging issues in mobile crowd sensing. Different studies are analyzed to identify the focus of mobile crowd sensing, its applications domains, privacy and security challenges and limitation for mobile crowd sensing with their possible solution. There is existing participant recruitment process is analyzed. We have identified few shortcomings in the mobile crowd sensing system is how to effectively identify and select the well-suited participants in recruitments from a large user pool and suggested selection algorithms that can improve the efficiency of the selection of the participants from the large user pool. The recommendations presented for the existing participant recruitment process that can improve the recruitment process, how we can recruit the well-suited participants from the large user pool.

Up to our best knowledge, this is the first work is done on the domain of MCS and the proposed designed modules to make the recruitment process efficient. These modules are a selection of ' $\mathrm{N}$ ' numbers of participant's recruitment algorithm. This algorithm efficiently recruits the participants and improves the performance of the algorithm. The selection of ' $\mathrm{N}$ ' number of participants recruitment algorithm with the goal of minimizing the sensing cost, while satisfying the certain level of coverage of mobile users. In future, we are interested to extend this research by developing energy efficient sensor device that will minimize the energy consumption and will increase the battery life of the smartphones.

\section{REFERENCES}

[1] Bellavista, P., et al. Human dynamics of mobile crowd sensing experimental datasets. in Communications (ICC), 2017 IEEE International Conference on. 2017. IEEE.

[2] Du, R., et al. Predicting activity attendance in event-based social networks: Content, context and social influence. in Proceedings of the 2014 ACM International Joint Conference on Pervasive and Ubiquitous Computing. 2014. ACM.

[3] Jaimes, L.G., I.J. Vergara-Laurens, and A. Raij, A survey of incentive techniques for mobile crowd sensing. IEEE Internet of Things Journal, 2015. 2(5): p. 370-380

[4] Ma, H., D. Zhao, and P. Yuan, Opportunities in mobile crowd sensing. IEEE Communications Magazine, 2014. 52(8): p. 29-35.

[5] Stansfeld, S.A. and M.P. Matheson, Noise pollution: non-auditory effects on health. British medical bulletin, 2003. 68(1): p. 243-257.

[6] Santini, S., B. Ostermaier, and A. Vitaletti. First experiences using wireless sensor networks for noise pollution monitoring. in Proceedings of the workshop on Real-world wireless sensor networks. 2008. ACM.

[7] Liu, C.H., et al., Energy-aware participant selection for smartphoneenabled mobile crowd sensing. IEEE Systems Journal, 2015.

[8] Narula, P., et al., MobileWorks: A Mobile Crowdsourcing Platform for Workers at the Bottom of the Pyramid. Human Computation, 2011. 11: p. 11.

[9] Kong, L., et al., Sustainable Incentive Mechanisms for Mobile Crowdsensing: Part 1. IEEE Communications Magazine, 2017. 55(3): p. 60-61.

[10] Pouryazdan, M., et al., Quantifying User Reputation Scores, Data Trustworthiness, and User Incentives in Mobile Crowd-Sensing. IEEE Access, 2017. 5: p. 1382-1397.

[11] Ren, J., et al., Exploiting mobile crowdsourcing for pervasive cloud services: challenges and solutions. IEEE Communications Magazine, 2015. 53(3): p. 98-105.

[12] Yang, K., et al., Security and privacy in mobile crowdsourcing networks: challenges and opportunities. IEEE Communications Magazine, 2015. 53(8): p. 75-81.

[13] Govindaraj, D., et al., MoneyBee: Towards enabling a ubiquitous, efficient, and easytouse mobile crowdsourcing service in the emerging market. Bell Labs Technical Journal, 2011. 15(4): p. 79-92.

[14] Ganti, R.K., F. Ye, and H. Lei, Mobile crowdsensing: current state and future challenges. IEEE Communications Magazine, 2011. 49(11).

[15] Pournajaf, L., et al., A survey on privacy in mobile crowd sensing task management. Dept. Math. Comput. Sci., Emory Univ., Atlanta, GA, USA, Tech. Rep. TR-2014-002, 2014.

[16] Krontiris, I., M. Langheinrich, and K. Shilton, Trust and privacy in mobile experience sharing: future challenges and avenues for research. IEEE Communications Magazine, 2014. 52(8): p. 50-55. 
[17] Yi, K., et al., Fast participant recruitment algorithm for large-scale Vehicle-based Mobile Crowd Sensing. Pervasive and Mobile Computing, 2017.

[18] Reddy, S., D. Estrin, and M. Srivastava, Recruitment framework for participatory sensing data collections, in Pervasive Computing. 2010, Springer. p. 138-155.

[19] Tuncay, G.S., G. Benincasa, and A. Helmy. Participant recruitment and data collection framework for opportunistic sensing: A comparative analysis. in Proceedings of the 8th ACM MobiCom workshop on Challenged networks. 2013. ACM.

[20] Chon, Y., et al. Understanding the coverage and scalability of place- centric crowdsensing. in Proceedings of the 2013 ACM international joint conference on Pervasive and ubiquitous computing. 2013. ACM.

[21] Xiong, H., et al., EMC 3: Energy-efficient data transfer in mobile crowdsensing under full coverage constraint. Mobile Computing, IEEE Transactions on, 2015. 14(7): p. 1355-1368.

[22] Zhao, D., H. Ma, and L. Liu, Energy-efficient opportunistic coverage for people-centric urban sensing. Wireless networks, 2014. 20(6): p. 14611476.

[23] Pournajaf, L., et al. Spatial task assignment for crowd sensing with cloaked locations. in Mobile Data Management (MDM), 2014 IEEE 15th International Conference on. 2014. IEEE. 